<Back to results | 1 of 1

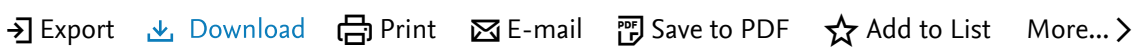

Full Text |View at Publisher|

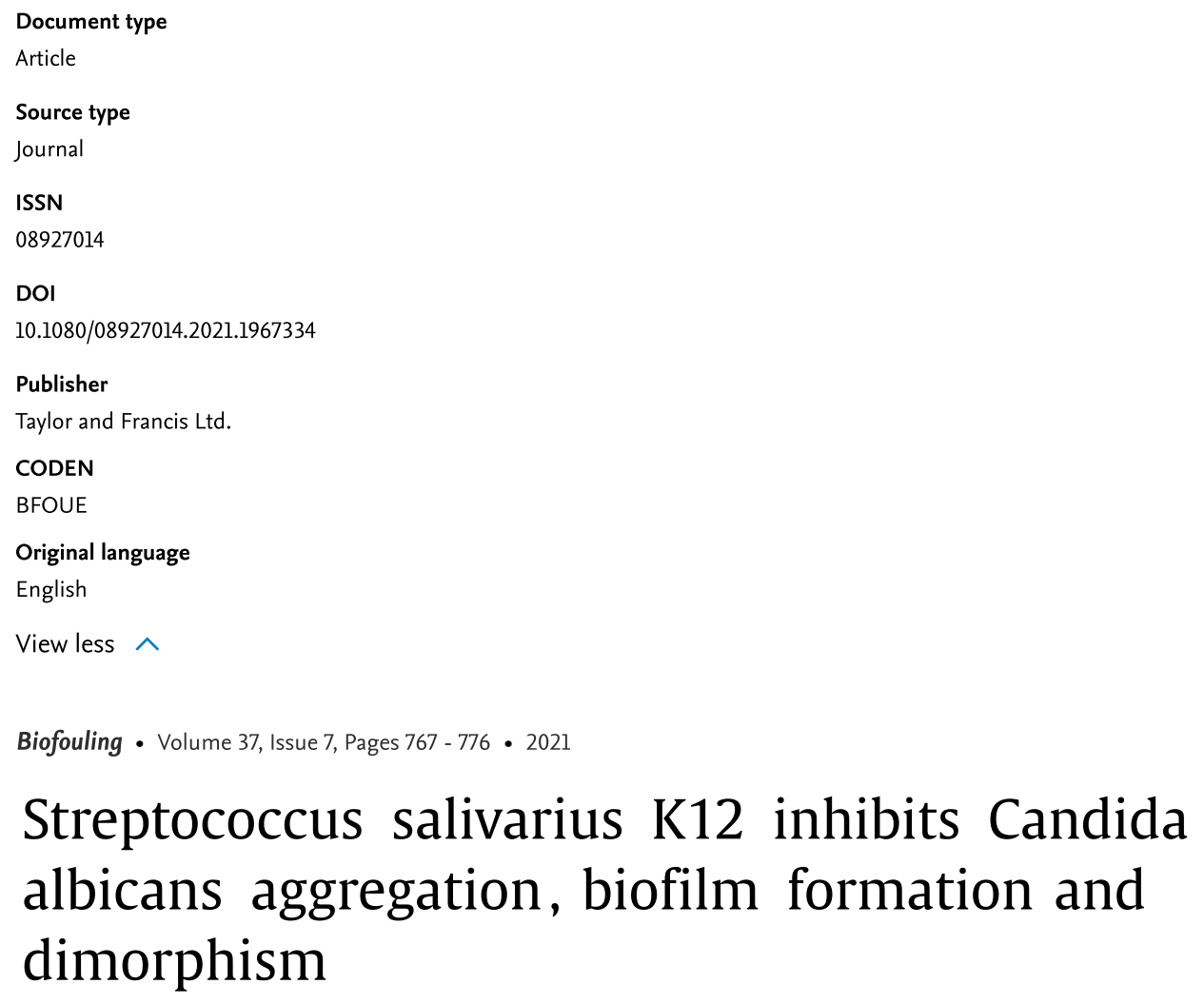

Biofouling • Volume 37, Issue 7, Pages $767-776 \cdot 2021$

\section{Streptococcus salivarius K12 inhibits Candida albicans aggregation, biofilm formation and dimorphism}

Mokhtar M. ${ }^{a}$ b, Rismayuddin N.A.R. ${ }^{b}, \quad$ Mat Yassim A.S. ${ }^{b, c}, \quad$ Ahmad H. ${ }^{a, b}, \quad$ Abdul Wahab R. ${ }^{a}, \quad$ Dashper S. ${ }^{d}$, Arzmi M.H. ${ }^{b, c}$

圆 Save all to author list

${ }^{a}$ Department of Biomedical Sciences, Kulliyyah of Allied Health Sciences, International Islamic University Malaysia, Kuantan, Pahang, Malaysia

${ }^{b}$ Cluster of Cancer Research Initiative IIUM (COCRII), International Islamic University Malaysia, Kuantan, Pahang, Malaysia

${ }^{\mathrm{C}}$ Department of Fundamental Dental and Medical Sciences, Kulliyyah of Dentistry, International Islamic University Malaysia, Kuantan, Pahang, Malaysia

${ }^{\mathrm{d}}$ Melbourne Dental School, The University of Melbourne, Melbourne, VIC, Australia

\section{Abstract}

Author keywords

Indexed keywords

SciVal Topics

Funding details

\section{Abstract}

Candida albicans causes candidiasis, particularly in immunocompromised patients. Streptococcus salivarius $\mathrm{K} 12$ ( K12) is a probiotic isolated from a healthy oral cavity. The study aimed to determine the effect of $\mathrm{K} 12$ on $\mathrm{C}$. albicans aggregation, biofilm formation and dimorphism.C. albicans
Cited by 0 documents

Inform me when this document is cited in Scopus:

Set citation alert >

\section{Related documents}

An in vitro model for oral mixed biofilms of Candida albicans and Streptococcus gordonii in synthetic saliva

Montelongo-Jauregui, D. , Srinivasan, A. ,

Ramasubramanian, A.K.

(2016) Frontiers in Microbiology

Candida-streptococcal interactions in biofilm-associated oral diseases

Koo, H. , Andes, D.R. , Krysan, D.J.

(2018) PLoS Pathogens

Interkingdom interaction between $\mathrm{C}$. albicans and $\mathrm{S}$. salivarius on titanium surfaces

Martorano-Fernandes, L. , Rodrigues, N.C. , de Souza Borges, M.H. (2020) BMC Oral Health

View all related documents based on references

Find more related documents in Scopus based on:

Authors > Keywords > 
ATCC MYA-4901, acquired immunodeficiency syndrome (AIDS) isolate (ALC2), and oral cancer isolate (ALC3) and K12 were used in the study. All C. albicans strains and K12 were grown in yeast peptone dextrose agar and brain heart infusion agar, respectively, prior to aggregation, biofilm and dimorphism assays. Auto-aggregation of C. albicans MYA-4901 and ALC2 was categorised as high, while the co-aggregation of the strains was low in the presence of $\mathrm{K} 12$. C. albicans total cell count decreased significantly when co-cultured with $\mathrm{K} 12$ compared with monocultured $\mathrm{C}$. albicans biofilm ( $\mathrm{p}$ <.05). Inhibition of yeast-to-hyphae transition was also observed when co-cultured with $\mathrm{K} 12$. In conclusion, K12 inhibits C. albicans aggregation, biofilm formation and dimorphism. (c) 2021 Informa UK Limited, trading as Taylor \& Francis Group.

Author keywords

aggregation; biofilm ; dimorphism ; Polymicrobial interactions; probiotic

Indexed keywords

SciVal Topics (i)

Funding details

References (64) View in search results format >

All

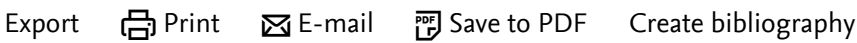

$1 \quad$ Allaker, R.P., Stephen, A.S.

Use of Probiotics and Oral Health (Open Access)

(2017) Current Oral Health Reports, 4 (4), pp. 309-318. Cited 37 times.

springer.com/journal/40496

doi: 10.1007/s40496-017-0159-6

View at Publisher

2 Alnuaimi, A.D., O'Brien-Simpson, N.M., Reynolds, E.C., Mccullough, M.J.

Clinical isolates and laboratory reference Candida species and strains have varying abilities to form biofilms (Open Access)

(2013) FEMS Yeast Research, 13 (7), pp. 689-699. Cited 45 times.

doi: 10.1111/1567-1364.12068

View at Publisher

3 Ardizzoni, A., Pericolini, E., Paulone, S., Orsi, C.F., Castagnoli, A., Oliva, I., Strozzi, E., (...), Blasi, E.

In vitro effects of commercial mouthwashes on several virulence traits of Candida albicans, viridans streptococci and Enterococcus faecalis colonizing the oral cavity (Open Access)

(2018) PLoS ONE, 13 (11), art. no. e0207262. Cited 15 times.

https://journals.plos.org/plosone/article/file?

id=10.1371/journal. pone.0207262\&type=printable

doi: $10.1371 /$ journal.pone.0207262

View at Publisher

4 Arzmi, M.H., Dashper, S., Catmull, D., Cirillo, N., Reynolds, E.S., McCullough, $M$.

Coaggregation of Candida albicans, Actinomyces naeslundii and Streptococcus mutans is Candida albicans strain dependent (Open Access)

(2015) FEMS Yeast Research, 15 (5). Cited 26 times. http://femsyr.oxfordjournals.org/ doi: $10.1093 /$ femsyr/fov038

View at Publisher 
5 Arzmi, M.H., Alnuaimi, A.D., Dashper, S., Cirillo, N., Reynolds, E.C., McCullough, M.

Polymicrobial biofilm formation by Candida albicans,

Actinomyces naeslundii, and Streptococcus mutans is

Candida albicans strain and medium dependent (Open Access)

(2016) Medical Mycology, 54 (8), pp. 856-864. Cited 16 times.

https://mmy.oxfordjournals.org/

doi: $10.1093 / \mathrm{mmy} / \mathrm{myw} 042$

View at Publisher

6 Bar-Yosef, H., Vivanco Gonzalez, N., Ben-Aroya, S., Kron, S.J., Kornitzer, D.

Chemical inhibitors of Candida albicans hyphal

morphogenesis target endocytosis (Open Access)

(2017) Scientific Reports, 7 (1), art. no. 5692. Cited 25 times.

www.nature.com/srep/index.html

doi: 10.1038/s41598-017-05741-y

View at Publisher

7 Burton, J.P., Wescombe, P.A., Moore, C.J., Chilcott, C.N., Tagg, J.R.

Safety assessment of the oral cavity probiotic Streptococcus salivarius K12 (Open Access)

(2006) Applied and Environmental Microbiology, 72 (4), pp. 3050-3053. Cited 101 times.

doi: 10.1128/AEM.72.4.3050-3053.2006

View at Publisher

8 Burton, J.P., Cowley, S., Simon, R.R., McKinney, J., Wescombe, P.A., Tagg, J.R. Evaluation of safety and human tolerance of the oral probiotic Streptococcus salivarius K12: A randomized, placebocontrolled, double-blind study

(2011) Food and Chemical Toxicology, 49 (9), pp. 2356-2364. Cited 50 times. doi: 10.1016/j.fct.2011.06.038

View at Publisher

9 Calderone, R.A., Fonzi, W.A.

Virulence factors of Candida albicans

(2001) Trends in Microbiology, 9 (7), pp. 327-335. Cited 920 times.

doi: 10.1016/S0966-842X(01)02094-7

View at Publisher

10 Cavalcanti, Y.W., Morse, D.J., da Silva, W.J., Del-Bel-Cury, A.A., Wei, X., Wilson, M., Milward, P., (...), Williams, D.W.

Virulence and pathogenicity of Candida albicans is enhanced in biofilms containing oral bacteria

(2015) Biofouling, 31 (1), pp. 27-38. Cited 57 times.

www.tandf.co.uk/journals/titles/08927014.asp

doi: $10.1080 / 08927014.2014 .996143$

View at Publisher 
11 Chukkapalli, S.S., Velsko, I.M., Rivera-Kweh, M.F., Zheng, D., Lucas, A.R., Kesavalu, L.

Polymicrobial oral infection with four periodontal bacteria orchestrates a distinct inflammatory response and atherosclerosis in ApoE ${ }^{\text {null }}$ mice (Open Access)

(2015) PLoS ONE, 10 (11), art. no. e0143291. Cited 48 times. http://www.plosone.org/article/fetchObject.action? uri=info:doi/10.1371/journal.pone.0143291\&representation=PDF doi: 10.1371/journal.pone.0143291

View at Publisher

12 Delorme, C., Abraham, A.-L., Renault, P., Guédon, E.

Genomics of Streptococcus salivarius, a major human commensal

(2015) Infection, Genetics and Evolution, 33, pp. 381-392. Cited 32 times. http://www.elsevier.com/locate/meegid doi: 10.1016/j.meegid.2014.10.001

View at Publisher

13 Denega, I., D'Enfert, C., Bachellier-Bassi, S.

Candida albicans biofilms are generally devoid of persister cells (Open Access)

(2019) Antimicrobial Agents and Chemotherapy, 63 (5), art. no. e01979-

18. Cited 7 times.

https://aac.asm.org/content/aac/63/5/e01979-18.full.pdf

doi: 10.1128/AAC.01979-18

View at Publisher

14 Dewhirst, F.E., Chen, T., Izard, J., Paster, B.J., Tanner, A.C.R., Yu, W.H., Lakshmanan, A., (...), Wade, W.G.

The human oral microbiome (Open Access)

(2010) Journal of Bacteriology, 192 (19), pp. 5002-5017. Cited 1649 times. http://jb.asm.org/cgi/reprint/192/19/5002 doi: 10.1128/JB.00542-10

View at Publisher

15 Dimopoulos, G., Paiva, J.-A., Meersseman, W., Pachl, J., Grigoras, I., Sganga, G., Montravers, P., (...), Ruhnke, M.

Efficacy and safety of anidulafungin in elderly, critically ill patients with invasive Candida infections: A post hoc analysis (Open Access)

(2012) International Journal of Antimicrobial Agents, 40 (6), pp. 521-

526. Cited 13 times.

doi: 10.1016/j.jijantimicag.2012.07.018

View at Publisher

16 Falsetta, M.L., Klein, M.I., Colonne, P.M., Scott-Anne, K., Gregoire, S., Pai, C.H., Gonzalez-Begne, M., (...), Koo, H.

Symbiotic relationship between Streptococcus mutans and Candida albicans synergizes virulence of plaque biofilms in vivo (Open Access)

(2014) Infection and Immunity, 82 (5), pp. 1968-1981. Cited 260 times.

http://iai.asm.org/content/82/5/1968.full.pdf

doi: 10.1128/IAI.00087-14

View at Publisher 
17 Ghannoum, M.A., Jurevic, R.J., Mukherjee, P.K., Cui, F., Sikaroodi, M., Naqvi, A., Gillevet, P.M.

Characterization of the oral fungal microbiome (mycobiome) in healthy individuals (Open Access)

(2010) PLoS Pathogens, 6 (1), art. no. el000713. Cited 578 times. http://www.plospathogens.org/article/fetchObjectAttachment.action? uri=info\%3Adoi\%2F10.1371\%2Fjournal.ppat.1000713\&representation=PDF doi: 10.1371/journal.ppat.1000713

View at Publisher

18 Gholizadeh, P., Eslami, H., Yousefi, M., Asgharzadeh, M., Aghazadeh, M., Kafil, H.S.

Role of oral microbiome on oral cancers, a review

(2016) Biomedicine and Pharmacotherapy, 84, pp. 552-558. Cited 91 times. www.elsevier.com/locate/biomedpharm doi: 10.1016/j.biopha.2016.09.082

View at Publisher

19 Hull, M.W., Chow, A.W.

Indigenous Microflora and Innate Immunity of the Head and Neck

(2007) Infectious Disease Clinics of North America, 21 (2), pp. 265-282. Cited 37 times.

doi: 10.1016/j.idc.2007.03.015

View at Publisher

20 Hyink, O., Wescombe, P.A., Upton, M., Ragland, N., Burton, J.P., Tagg, J.R.

Salivaricin $A 2$ and the novel lantibiotic salivaricin $B$ are encoded at adjacent loci on a 190-kilobase transmissible megaplasmid in the oral probiotic strain Streptococcus salivanus K12 (Open Access)

(2007) Applied and Environmental Microbiology, 73 (4), pp. 1107-1113. Cited 103 times.

doi: 10.1128/AEM.02265-06

View at Publisher

21 Ishijima, S.A., Hayama, K., Burton, J.P., Reid, G., Okada, M., Matsushita, Y., Abe, $S$.

Effect of Streptococcus salivarius K12 on the In vitro growth of Candida albicans and its protective effect in an oral candidiasis model (Open Access)

(2012) Applied and Environmental Microbiology, 78 (7), pp. 2190-2199. Cited 68 times.

http://aem.asm.org/content/78/7/2190.full.pdf doi: 10.1128/AEM.07055-11

View at Publisher

22 Ishikawa, K.H., Mayer, M.P.A., Miyazima, T.Y., Matsubara, V.H., Silva, E.G., Paula, C.R., Campos, T.T., (...), Nakamae, A.E.M.

A multispecies probiotic reduces oral candida colonization in denture wearers

(2015) Journal of Prosthodontics, 24 (3), pp. 194-199. Cited 56 times. doi: $10.1111 /$ jopr.12198

View at Publisher 
23 Jabra-Rizk, M.A., Kong, E.F., Tsui, C., Nguyen, M.H., Clancy, C.J., Fidel, P.L., Noverr, M.

Candida albicans pathogenesis: Fitting within the hostmicrobe damage response framework (Open Access)

(2016) Infection and Immunity, 84 (10), pp. 2724-2739. Cited 87 times. http://iai.asm.org/content/84/10/2724.full.pdf doi: 10.1128/IAI.00469-16

View at Publisher

24 Jakubovics, N.S.

Intermicrobial Interactions as a Driver for Community Composition and Stratification of Oral Biofilms

(2015) Journal of Molecular Biology, 427 (23), pp. 3662-3675. Cited 48 times. https://www.journals.elsevier.com/journal-of-molecular-biology. doi: 10.1016/j.jmb.2015.09.022

View at Publisher

25 James, K.M., MacDonald, K.W., Chanyi, R.M., Cadieux, P.A., Burton, J.P.

Inhibition of Candida albicans biofilm formation and modulation of gene expression by probiotic cells and supernatant (Open Access)

(2016) Journal of Medical Microbiology, 65 (4), pp. 328-336. Cited 36 times. http://www.microbiologyresearch.org/docserver/fulltext/jmm/65/4/320_jmm0 00231.pdf?expires

doi: $10.1099 / \mathrm{jmm} .0 .000226$

View at Publisher

26 Jarosz, L.M., Deng, D.M., Van Der Mei, H.C., Crielaard, W., Krom, B.P. Streptococcus mutans competence-stimulating peptide inhibits candida albicans hypha formation (Open Access)

(2009) Eukaryotic Cell, 8 (11), pp. 1658-1664. Cited 121 times.

http://ec.asm.org/cgi/reprint/8/11/1658

doi: 10.1128/EC.00070-09

View at Publisher

27 Kaci, G., Goudercourt, D., Dennin, V., Pot, B., Doré, J., Ehrlich, S.D., Renault, P., (...), Delorme, C.

Anti-inflammatory properties of Streptococcus salivarius, a commensal bacterium of the oral cavity and digestive tract (Open Access)

(2014) Applied and Environmental Microbiology, 80 (3), pp. 928-934. Cited 59 times.

http://aem.asm.org/content/80/3/928.full.pdf

doi: 10.1128/AEM.03133-13

View at Publisher

28 Kauffman, C.A.

Fungal infections in older adults (Open Access)

(2001) Clinical Infectious Diseases, 33 (4), pp. 550-555. Cited 94 times. doi: $10.1086 / 322685$

View at Publisher 
29 Klotz, S.A., Gaur, N.K., De Armond, R., Sheppard, D., Khardori, N., Edwards, J.E., Lipke, P.N., (...), El-Azizi, M.

Candida albicans Als proteins mediate aggregation with bacteria and yeasts (Open Access)

(2007) Medical Mycology, 45 (4), pp. 363-370. Cited 82 times. doi: $10.1080 / 13693780701299333$

View at Publisher

30 Koh, A.Y., Köhler, J.R., Coggshall, K.T., Van Rooijen, N., Pier, G.B.

Mucosal damage and neutropenia are required for Candida albicans dissemination (Open Access)

(2008) PLoS Pathogens, 4 (2). Cited 228 times.

http://www.plospathogens.org/article/fetchObjectAttachment.action? uri=info\%3Adoi\%2F10.1371\%2Fjournal.ppat.0040035\&representation=PDF doi: 10.1371/journal.ppat.0040035

View at Publisher

31 Kong, E.F., Kucharíková, S., Van Dijck, P., Peters, B.M., Shirtliff, M.E., JabraRizka, M.A.

Clinical implications of oral candidiasis: Host tissue damage and disseminated bacterial disease (Open Access)

(2015) Infection and Immunity, 83 (2), pp. 604-613. Cited 58 times. http://iai.asm.org/content/83/2/604.full.pdf doi: 10.1128/IAI.02843-14

View at Publisher

32 Krom, B.P., Kidwai, S., Ten Cate, J.M.

Candida and other fungal species: Forgotten players of healthy oral microbiota

(2014) Journal of Dental Research, 93 (5), pp. 445-451. Cited 72 times.

http://jdr.sagepub.com/

doi: $10.1177 / 0022034514521814$

View at Publisher

33 LaFleur, M.D., Kumamoto, C.A., Lewis, K.

Candida albicans biofilms produce antifungal-tolerant persister cells (Open Access)

(2006) Antimicrobial Agents and Chemotherapy, 50 (11), pp. 3839-3846. Cited 336 times.

doi: 10.1128/AAC.00684-06

View at Publisher

34 Lohse, M.B., Gulati, M., Johnson, A.D., Nobile, C.J.

Development and regulation of single-and multi-species Candida albicans biofilms (Open Access)

(2018) Nature Reviews Microbiology, 16 (1), pp. 19-31. Cited 187 times. http://www.nature.com/nrmicro/index.html doi: $10.1038 /$ nrmicro.2017.107

View at Publisher

35 Lone, M.S., Bashir, G., Bali, N., Sajad, S., Aejaz, S., Bashir, H., Ahmad, J. Oral Candida colonization and infection in cancer patients and their antifungal susceptibility in a tertiary care hospital (2014) Int J Adv Res, 2, pp. 541-550. Cited 11 times. 
36 Low, C.-Y., Rotstein, C.

Emerging fungal infections in immunocompromised patients (Open Access)

(2011) F1000 Medicine Reports, 3 (1), art. no. 14. Cited 169 times. http://f1000.com/reports/m/3/14/pdf doi: 10.3410/M3-14

View at Publisher

37 McCall, A.D., Pathirana, R.U., Prabhakar, A., Cullen, P.J., Edgerton, M.

Candida albicans biofilm development is governed by cooperative attachment and adhesion maintenance proteins (Open Access)

(2019) npj Biofilms and Microbiomes, 5 (1), art. no. 21. Cited 30 times. https://www.nature.com/npjbiofilms/ doi: 10.1038/s41522-019-0094-5

View at Publisher

38 Montelongo-Jauregui, D., Lopez-Ribot, J.L.

Candida interactions with the oral bacterial microbiota (Open Access)

(2018) Journal of Fungi, 4 (4), art. no. 122. Cited 16 times.

www.mdpi.com/journal/jof

doi: $10.3390 /$ jof 4040122

View at Publisher

39 Morales, D.K., Hogan, D.A.

Candida albicans interactions with bacteria in the context of human health and disease (Open Access)

(2010) PLoS Pathogens, 6 (4), pp. 1-4. Cited 177 times.

http://www.plospathogens.org/article/fetchObjectAttachment.action? uri=info\%3Adoi\%2F10.1371\%2Fjournal.ppat. 1000886\&representation=PDF doi: 10.1371/journal.ppat.1000886

View at Publisher

40 Nobbs, A.H., Jenkinson, H.F.

Interkingdom networking within the oral microbiome (Open Access)

(2015) Microbes and Infection, 17 (7), pp. 484-492. Cited 26 times. http://www.journals.elsevier.com/microbes-and-infection/ doi: $10.1016 /$ j.micinf.2015.03.008

View at Publisher

41 Nobile, C.J., Johnson, A.D.

Candida albicans Biofilms and Human Disease (Open Access)

(2015) Annual Review of Microbiology, 69 (1), pp. 71-92. Cited 441 times. http://arjournals.annualreviews.org/loi/micro doi: 10.1146/annurev-micro-091014-104330

View at Publisher 
42 Pearce, C., Bowden, G.H., Evans, M., Fitzsimmons, S.P., Johnson, J., Sheridan, M.J., Wientzen, R., (...), Cole, M.F.

Identification of pioneer viridans streptococci in the oral cavity of human neonates (Open Access)

(1995) Journal of Medical Microbiology, 42 (1), pp. 67-72. Cited 96 times. http://jmm.sgmjournals.org/

doi: 10.1099/00222615-42-1-67

View at Publisher

43 Di Pierro, F., Colombo, M., Zanvit, A., Risso, P., Rottoli, A.S.

Use of Streptococcus salivarius $\mathrm{K} 12$ in the prevention of streptococcal and viral pharyngotonsillitis in children (Open Access)

(2014) Drug, Healthcare and Patient Safety, 6 (1), pp. 15-20. Cited 38 times. http://www.dovepress.com/getfile.php?fileID=18986 doi: $10.2147 /$ dhps.559665

View at Publisher

44 Prakash, B., Shekar, M., Maiti, B., Karunasagar, I., Padiyath, S.

Prevalence of candida spp. Among healthy denture and nondenture wearers with respect to hygiene and age (Open Access)

(2015) Journal of Indian Prosthodontist Society, 15 (1), pp. 29-32. Cited 16 times.

http://www.springer.com/medicine/dentistry/journal/13191 doi: 10.4103/0972-4052.155041

View at Publisher

45 Rodrigues, M.E., Gomes, F., Rodrigues, C.F.

Candida spp./bacteria mixed biofilms (Open Access)

(2020) Journal of Fungi, 6 (1), art. no. 5. Cited 32 times.

https://www.mdpi.com/2309-608X/6/1/5/pdf

doi: $10.3390 /$ jof6010005

View at Publisher

46 Sato, T., Kishi, M., Suda, M., Sakata, K., Shimoda, H., Miura, H., Ogawa, A., (...), Kobayashi, $S$.

Prevalence of Candida albicans and non-albicans on the tongue dorsa of elderly people living in a post-disaster area: A cross-sectional survey (Open Access)

(2017) BMC Oral Health, 17 (1), art. no. 51. Cited 11 times. http://www.biomedcentral.com/bmcoralhealth/ doi: $10.1186 / \mathrm{s} 12903-017-0342-0$

View at Publisher

47 Silverman, R.J., Nobbs, A.H., Vickerman, M.M., Barbour, M.E., Jenkinson, H.F.

Interaction of Candida albicans cell wall Als3 Protein with Streptococcus gordonii SspB adhesin promotes development of mixed-species communities (Open Access)

(2010) Infection and Immunity, 78 (11), pp. 4644-4652. Cited 150 times. http://iai.asm.org/cgi/reprint/78/11/4644

doi: 10.1128/IAI.00685-10

View at Publisher 
48 Smith, D.J., Anderson, J.M., King, W.F., van Houte, J., Taubman, M.A.

Oral streptococcal colonization of infants

(1993) Oral Microbiology and Immunology, 8 (1), pp. 1-4. Cited 78 times. doi: 10.1111/j.1399-302X.1993.tb00535.x

View at Publisher

49 Southey-Pillig, C.J., Davies, D.G., Sauer, K.

Characterization of temporal protein production in

Pseudomonas aeruginosa biofilms (Open Access)

(2005) Journal of Bacteriology, 187 (23), pp. 8114-8126. Cited 151 times. doi: 10.1128/JB.187.23.8114-8126.2005

View at Publisher

50 Staniszewska, $M$

Virulence factors in candida species

(2020) Current Protein and Peptide Science, 21 (3), pp. 313-323. Cited 14

times.

http://www.eurekaselect.com/173711/article

doi: 10.2174/1389203720666190722152415

View at Publisher

51 Su, C., Yu, J., Lu, Y.

Hyphal development in Candida albicans from different cell states

(2018) Current Genetics, 64 (6), pp. 1239-1243. Cited 17 times.

link.springer.de/link/service/journals/00294/index.htm

doi: 10.1007/s00294-018-0845-5

View at Publisher

52 Taylan, I., Özcan, I., Mumcuoǧlu, I., Baran, I., Özcan, K.M., Akdoǧan, O., Selcuk, A., (...), Dere, H.

Comparison of the Surface and Core Bacteria in Tonsillar and Adenoid Tissue With Beta-Lactamase Production (Open Access)

(2011) Indian Journal of Otolaryngology and Head and Neck Surgery, 63 (3), pp. 223-228. Cited 19 times. doi: $10.1007 /$ s12070-011-0265-z

View at Publisher

53 Urban, C.F., Reichard, U., Brinkmann, V., Zychlinsky, A.

Neutrophil extracellular traps capture and kill Candida albicans and hyphal forms (Open Access)

(2006) Cellular Microbiology, 8 (4), pp. 668-676. Cited 623 times. doi: 10.1111/j.1462-5822.2005.00659.x

View at Publisher

54 Vila, T., Sultan, A.S., Montelongo-Jauregui, D., Jabra-Rizk, M.A.

Oral candidiasis: A disease of opportunity (Open Access)

(2020) Journal of Fungi, 6 (1), art. no. 15. Cited 46 times.

https://www.mdpi.com/2309-608X/6/1/15/pdf

doi: $10.3390 /$ jof6010015

View at Publisher 
55 Vílchez, R., Lemme, A., Ballhausen, B., Thiel, V., Schulz, S., Jansen, R., Sztajer, H., (...), Wagner-Döbler, I.

Streptococcus mutans inhibits Candida albicans hyphal formation by the fatty acid signaling molecule trans-2decenoic acid (SDSF)

(2010) ChemBioChem, 11 (11), pp. 1552-1562. Cited 84 times.

http://www3.interscience.wiley.com/cgi-bin/fulltext/123549118/PDFSTART doi: 10.1002/cbic.201000086

View at Publisher

56 Wall, G., Montelongo-Jauregui, D., Vidal Bonifacio, B., Lopez-Ribot, J.L., Uppuluri, P.

Candida albicans biofilm growth and dispersal: contributions to pathogenesis (Open Access)

(2019) Current Opinion in Microbiology, 52, pp. 1-6. Cited 44 times. http://www.elsevier.com/locate/mib doi: 10.1016/j.mib.2019.04.001

View at Publisher

57 Weerasekera, M.M., Wijesinghe, G.K., Jayarathna, T.A., Gunasekara, C.P., Fernando, N., Kottegoda, N., Samaranayake, L.P.

Culture media profoundly affect Candida Albicans and Candida tropicalis growth, adhesion and biofilm development (Open Access)

(2016) Memorias do Instituto Oswaldo Cruz, 111 (11), pp. 697-702. Cited 41 times.

http://memorias.ioc.fiocruz.br/issues/currentissue/item/download/7625_d7c5f33b074139bb760dld52ca063943 doi: 10.1590/0074-02760160294

View at Publisher

58 Williams, D., Lewis, M.

Pathogenesis and treatment of oral candidosis (Open Access)

(2011) Journal of Oral Microbiology, 3 (2011), art. no. 5771. Cited 185 times. http://www.journaloforalmicrobiology.net/index.php/jom/article/download/57 71/6961

doi: $10.3402 / j o m . v 3 i 0.5771$

View at Publisher

$59 \mathrm{Xu}, \mathrm{H}$., Jenkinson, H.F., Dongari-Bagtzoglou, A.

Innocent until proven guilty: Mechanisms and roles of Streptococcus-Candida interactions in oral health and disease (Open Access)

(2014) Molecular Oral Microbiology, 29 (3), pp. 99-116. Cited 71 times. http://www.interscience.wiley.com/jpages/2041-1006 doi: 10.1111/omi.12049

View at Publisher

60 Yang, Y.-L.

Virulence factors of Candida species

(2003) Journal of Microbiology, Immunology and Infection, 36 (4), pp. 223 228. Cited 172 times. 
61 Yoo, H.-J., Jwa, S.-K., Kim, D.-H., Ji, Y.-J.

Inhibitory effect of Streptococcus salivarius $\mathrm{K} 12$ and $\mathrm{M} 18$ on halitosis in vitro (Open Access)

(2020) Clinical and Experimental Dental Research, 6 (2), pp. 207-214. Cited 3 times.

www.cedrjournal.com

doi: $10.1002 / \mathrm{cre} 2.269$

View at Publisher

62 Zago, C.E., Silva, S., Sanitá, P.V., Barbugli, P.A., Dias, C.M.I., Lordello, V.B., Vergani, C.E.

Dynamics of biofilm formation and the Interaction between Candida albicans and methicillin-susceptible (MSSA) and resistant Staphylococcus aureus (MRSA) (Open Access)

(2015) PLoS ONE, 10 (4), art. no. e0123206. Cited 77 times.

http://www.plosone.org/article/fetchObject.action?

uri=info:doi/10.1371/journal.pone.0123206\&representation=PDF

doi: 10.1371/journal.pone.0123206

View at Publisher

63 Zijnge, V., Van Leeuwen, M.B.M., Degener, J.E., Abbas, F., Thurnheer, T., Gmür, R., Harmsen, H.J.M.

Oral biofilm architecture on natural teeth (Open Access)

(2010) PLoS ONE, 5 (2), art. no. e9321. Cited 371 times.

http://www.plosone.org/article/fetchObjectAttachment.action? uri=info\%3Adoi\%2F10.1371\%2Fjournal.pone.0009321\&representation=PDF doi: 10.1371/journal.pone.0009321

View at Publisher

64 Zupancic, K., Kriksic, V., Kovacevic, I., Kovacevic, D.

Influence of Oral Probiotic Streptococcus salivarius K12 on Ear and Oral Cavity Health in Humans: Systematic Review

(2017) Probiotics and Antimicrobial Proteins, 9 (2), pp. 102-110. Cited 24 times.

http://www.springer.com/new+\%26+forthcoming+titles+\%28default\%29/jour nal/12602

doi: $10.1007 /$ s12602-017-9261-2

View at Publisher

ㅇ Arzmi, M.H.; Cluster of Cancer Research Initiative IIUM (COCRII), International Islamic University Malaysia, Kuantan, Pahang, Malaysia

(C) Copyright 2021 Elsevier B.V., All rights reserved.

\section{About Scopus}

What is Scopus

Content coverage

Scopus blog

Scopus API

Privacy matters

\section{Language}

日本語に切り替える

切换到简体中文

切換到繁體中文

Русский язык
Customer Service

Help

Contact us 

use of cookies. 